Supporting Information

\title{
Achieving an Accurate Surface Profile of a Photonic Crystal for Near-Unity Solar Absorption in a Super Thin-Film Architecture
}

Ping Kuang, ${ }^{\dagger}$ Sergey Eyderman, ${ }^{\ddagger}$ Mei-Li Hsieh, ${ }^{\ddagger}$ Anthony Post, ${ }^{\dagger}$ Sajeev John, ${ }^{\ddagger}$ and Shawn-Yu $\operatorname{Lin}{ }^{* \dagger}$

${ }^{\dagger}$ The Future Chips Constellation and the Department of Physics, Applied Physics, and Astronomy, Rensselaer Polytechnic Institute, 110 Eighth Street, Troy, New York 12180, United States

${ }^{\ddagger}$ Department of Physics, University of Toronto, 60 St. George Street, Toronto, Ontario M5S 1A7, Canada

${ }^{*}$ Department of Photonics, National Chiao-Tung University, Hsinchu, Taiwan 300

Electronic mail: sylin@rpi.edu, kuangp2@rpi.edu 


\section{Supporting Figures and table:}

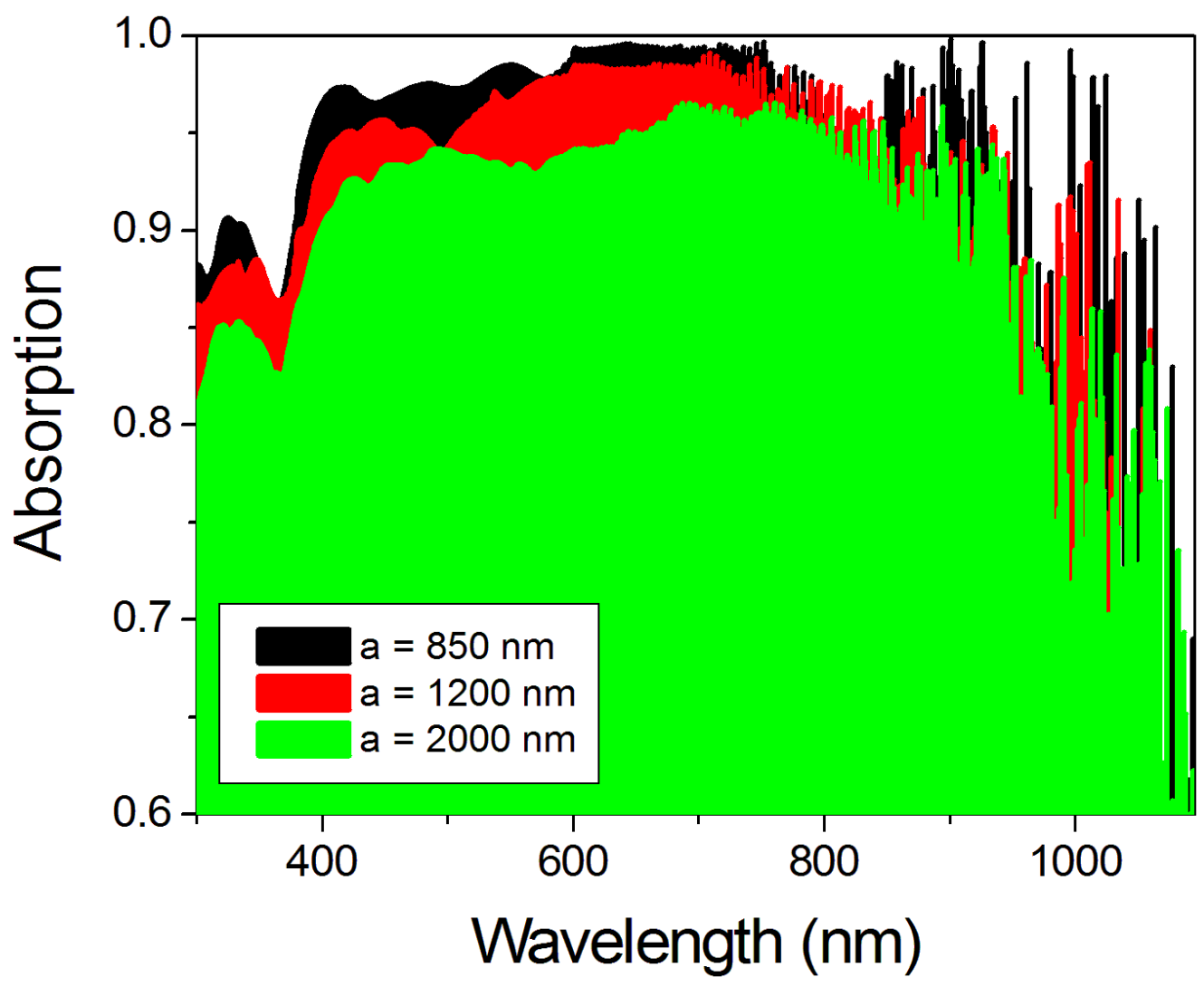

Figure S1. Dependence of absorption on the periodicity of Teepee-like photonic crystals.

Our FDTD simulation shows that the absorption is dependent on the periodicity of Teepee-like photonic crystals. The height of the photonic crystal is also changed according to the constant $h /(a / 2)$ ratio $=2.3$ as mentioned in the main text. It is clearly shown that, as the periodicity decreases, the absorption is increased in both shorter and longer wavelength ranges, respectively. The optimal periodicity is around $850 \mathrm{~nm}$. For the shorter wavelength range $(\lambda=300-850 \mathrm{~nm})$, the absorption increase is due to better index matching between passivation oxide layer $(\mathrm{t}=60$ $\mathrm{nm})$ and the photonic crystal. For the longer wavelength range $(\lambda=850-1100 \mathrm{~nm})$, the absorption enhancement is due to the parallel-to-interface refraction effect, which is shown as multiple absorption peaks. It is clear that the intensity of these peaks are increased as the periodicity is reduced to $850 \mathrm{~nm}$, indicating the PIR light trapping effect has become more dominant in this wavelength range as well. 


\begin{tabular}{|c|c|c|c|c|}
\hline & \multicolumn{2}{|c|}{$\begin{array}{c}\text { Near-IR } \\
(\lambda=\mathbf{8 5 0 - 1 1 0 0 n m})\end{array}$} & \multicolumn{2}{c|}{$\begin{array}{c}\text { Visible + Near-IR } \\
(\lambda=300-1100 n m)\end{array}$} \\
\hline $\begin{array}{c}\text { Periodicity } \\
(\mathrm{nm})\end{array}$ & $\begin{array}{c}J_{\text {Lambertian }^{2}} \\
\left(\mathrm{~mA} / \mathrm{cm}^{2}\right)\end{array}$ & $J_{P C}\left({\left.\mathrm{~mA} / \mathrm{cm}^{2}\right)}^{2}\right.$ & $\begin{array}{c}J_{\text {Lambertian }} \\
\left(\mathrm{mA} / \mathrm{cm}^{2}\right)\end{array}$ & $J_{P C}\left(\mathrm{~mA} / \mathrm{cm}^{2}\right)$ \\
\hline 550 & 9.3 & 11.9 & 39.5 & 38.9 \\
\hline 850 & 9.3 & 12.2 & 39.5 & 39.7 \\
\hline 1200 & 9.3 & 12.0 & 39.5 & 39.1 \\
\hline 2000 & 9.3 & 11.6 & 39.5 & 38.9 \\
\hline
\end{tabular}

Table S1. Dependence of Maximum achievable photocurrent density (MAPD) on the periodicity of Teepee-like photonic crystals for near-IR $(\lambda=850-1100 \mathrm{~nm})$ and visible + near-IR $(\lambda=300$ $1100 \mathrm{~nm}$ ) wavelength ranges, respectively.

The MAPD was calculated for near-IR $(\lambda=850-1100 \mathrm{~nm})$ and visible and near-IR $(\lambda=300-1100$ $\mathrm{nm}$ ) wavelength ranges, respectively, following reference 38 in the main text. In addition, the MAPD in both ranges are calculated for Lambertian limit:

$\mathrm{A}=1-\frac{1}{1+\alpha L_{\text {trap }}}$

Here, $L_{\text {trap }}=4 n^{2} L, n=3.5$ is the real part of refractive index of silicon, $\alpha=4 \pi k / \lambda$ is the absorption coefficient (where $\mathrm{k}$ is an imaginary part of the refractive index of silicon), and $\mathrm{L}$ is the thickness of silicon. It is shown that, in the near-IR range $(\lambda=850-1100 \mathrm{~nm})$, all of the photonic crystal structures outperform the Lambertian limit, with the best MAPD obtained by the PC structure with periodicity a $=850 \mathrm{~nm}$. The fixed passivation oxide layer thickness $(t=60 \mathrm{~nm})$ reduces the absorption in the visible $(\lambda=300-850 \mathrm{~nm})$ wavelength range for photonic crystals with other lattice constants, but optimal tradeoff between light trapping and antireflection for PC structure with periodicity a $=850 \mathrm{~nm}$ shows the MAPD reaches its maximum at $39.7 \mathrm{~mA} / \mathrm{cm}^{2}$, which is slightly higher than the Lambertian limit. (see reference 21 and 25 in main text) 


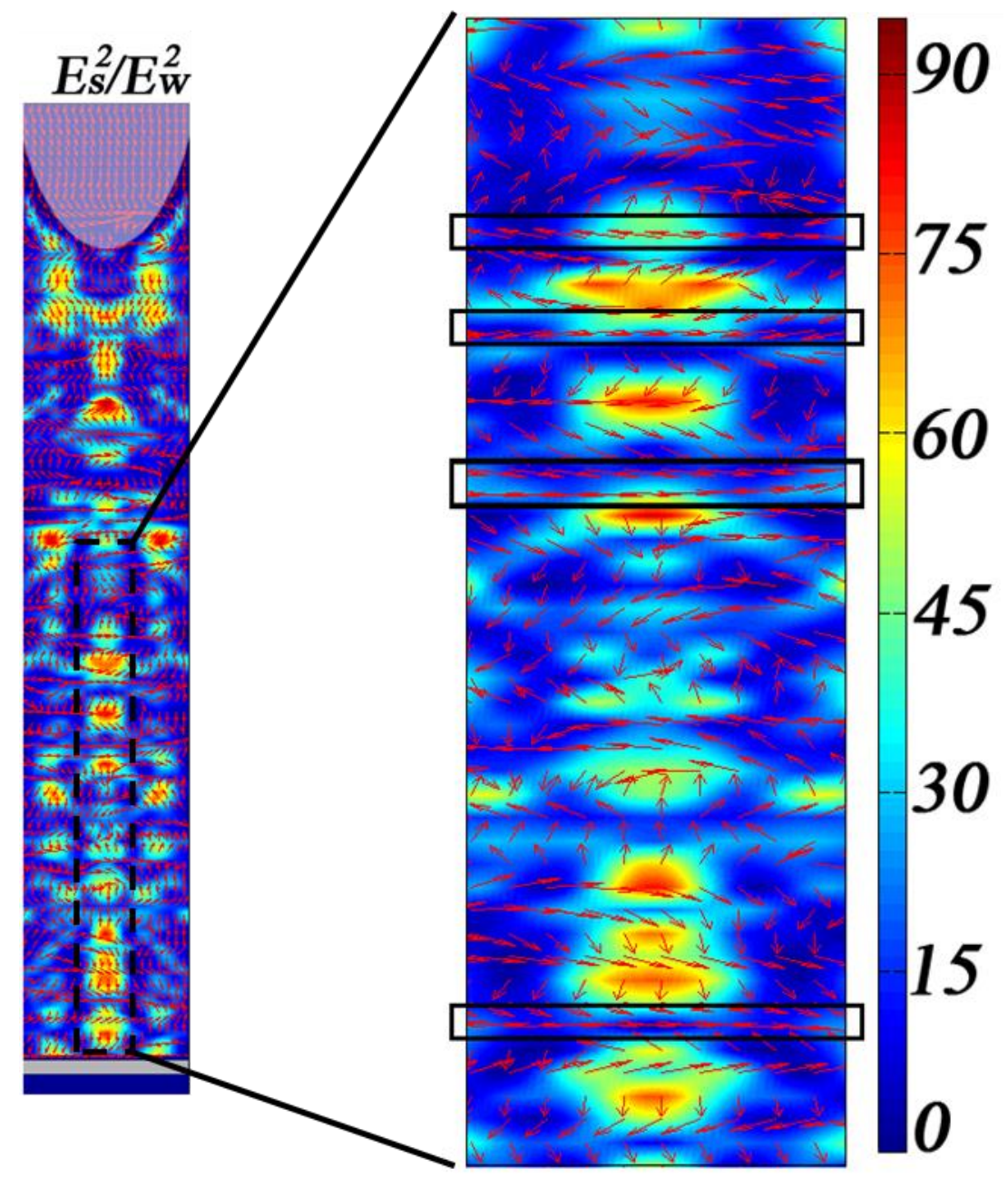

Figure S2. Orthogonal slice for resonance at $\lambda=1070 \mathrm{~nm}$ for Poynting vector and energy density distribution shows Vortex-like, high field concentration in a $10 \mu \mathrm{m}$ photonic crystal silicon film with sunlight impinging from the top. The zoomed-in, high resolution section of the energy density distribution plot shows the close-up of the high field concentration regions. (red color regions). In addition, the Poynting vector exhibits vortex-like circulation patterns. This circulation and almost parallel-to-interface energy flow (red arrows) enhances the dwell time of light in the cell, leading to a substantial absorption increase in the near infrared region where the intrinsic absorption of silicon is weak. 


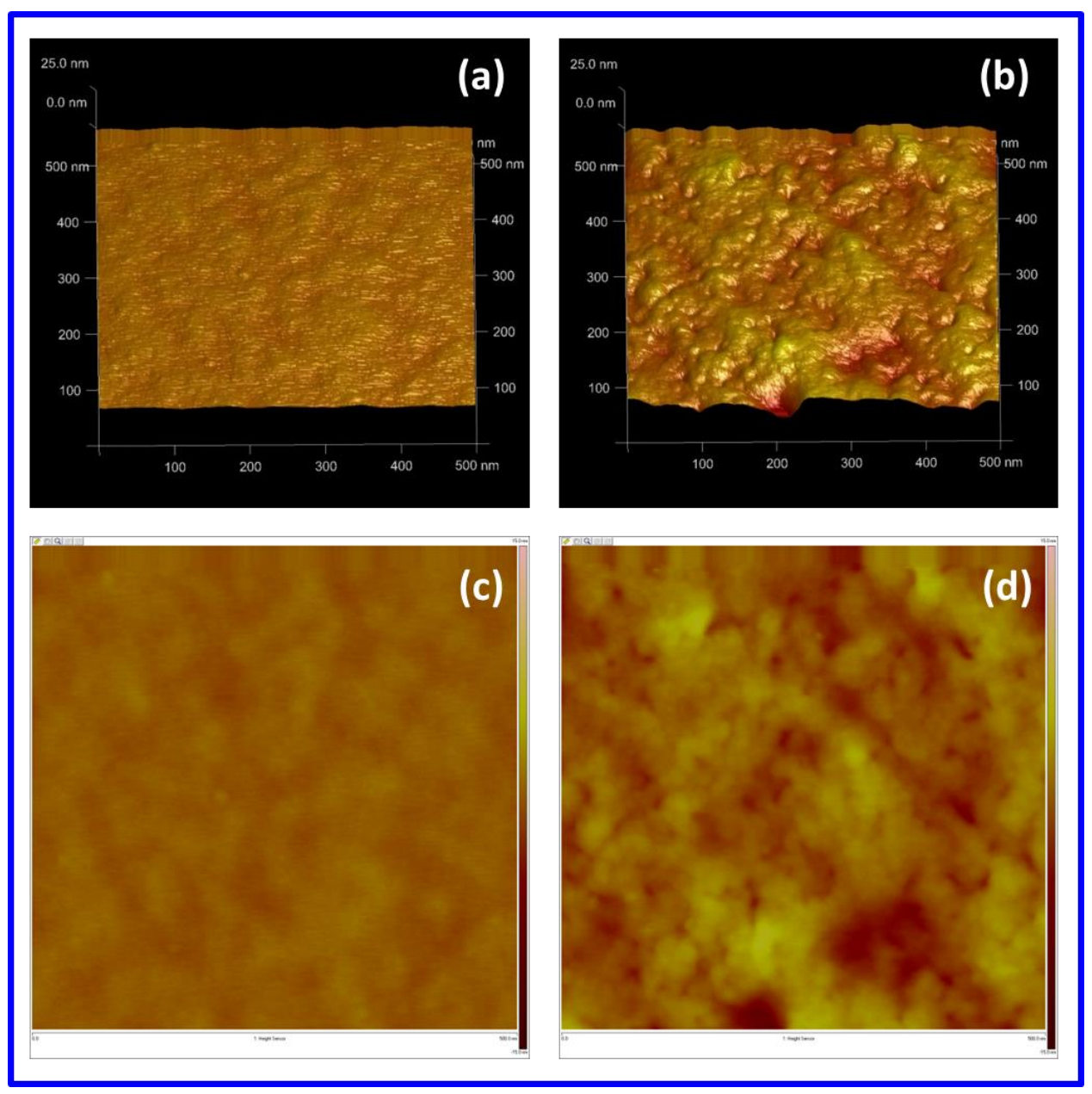

Figure S3. AFM characterizations of flat silicon with or without RIE etching. (a) 3D rendering and (c) 2D image of flat silicon without RIE etching, which has a root mean square (RMS) roughness $\left(\mathrm{R}_{\mathrm{q}}\right)$ of $0.45 \mathrm{~nm}$. (b) 3D image and (d) 2D image of flat silicon with RIE etching, which has a root mean square (RMS) roughness $\left(\mathrm{R}_{\mathrm{q}}\right)$ of $1.82 \mathrm{~nm}$. All images were scanned with the same scan area $(500 \mathrm{~nm} X 500 \mathrm{~nm})$ and vertical range $(Z=25 \mathrm{~nm})$. 


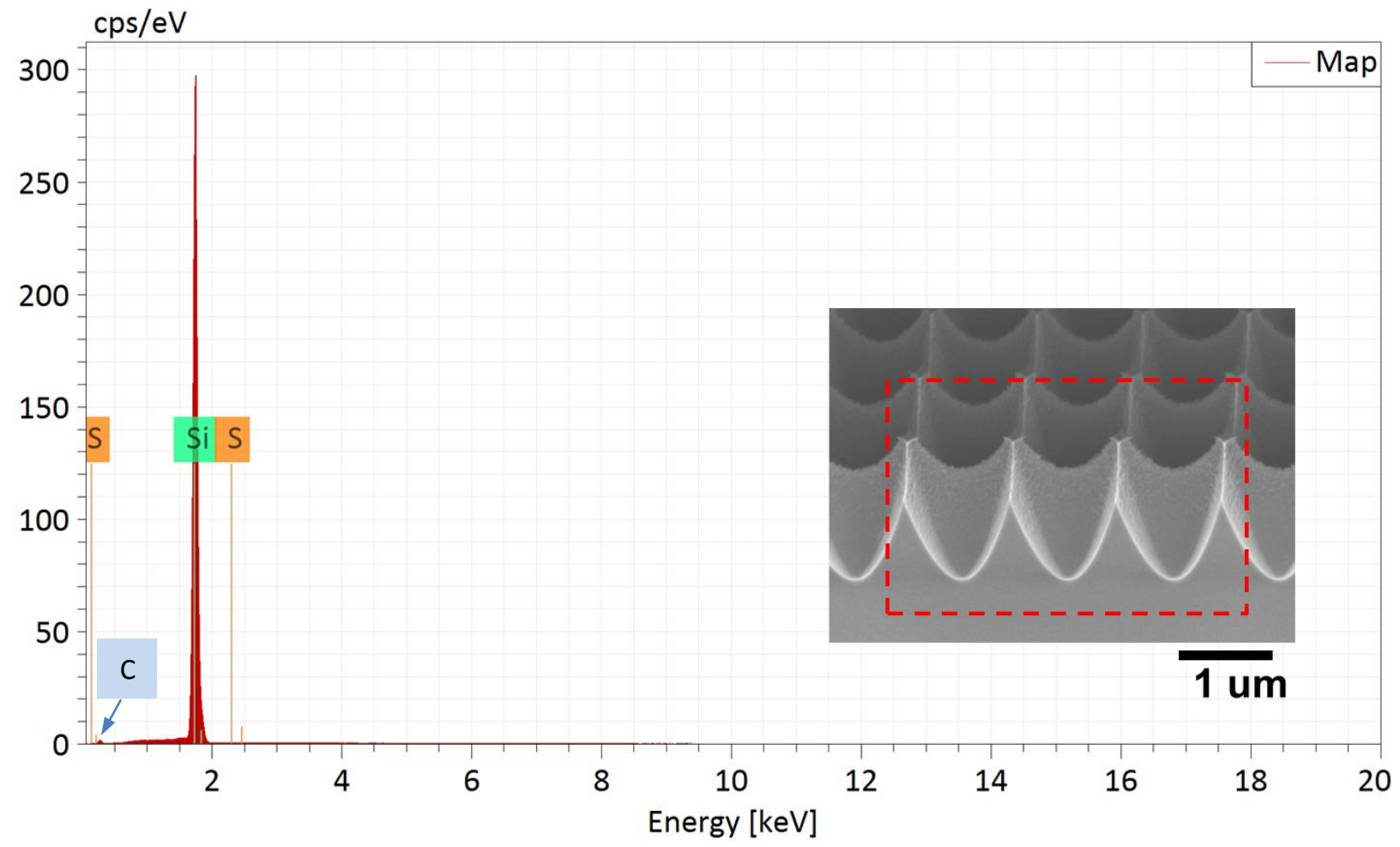

Figure S4. Energy-dispersive X-ray Spectrometer (EDS) analysis of Tee-pee PC structure with RIE etching. The scanned area is within the red rectangle of the SEM image. It is clear that the silicon peak is dominant. Some carbon was detected, but no noticeable sulfur is detected. 